This is the author's final, peer-reviewed manuscript as accepted for publication. The publisher-formatted version may be available through the publisher's web site or your institution's library.

\title{
Genetic characterization and phylogenetic analysis of skunk- associated rabies viruses in North America with special emphasis on the central plains
}

Rolan Davis, Susan A. Nadin-Davis, Michael Moore and Cathleen Hanlon

\section{How to cite this manuscript}

If you make reference to this version of the manuscript, use the following information:

Davis, R., Nadin-Davis, S. A., Moore, M., \& Hanlon, C. (2013). Genetic characterization and phylogenetic analysis of skunk-associated rabies viruses in North America with special emphasis on the central plains. Retrieved from http://krex.ksu.edu

\section{Published Version Information}

Citation: Davis, R., Nadin-Davis, S. A., Moore, M., \& Hanlon, C. (2013). Genetic characterization and phylogenetic analysis of skunk-associated rabies viruses in North America with special emphasis on the central plains. Virus Research, 174(1-2), 27-36.

Copyright: @ 2013 Elsevier B.V.

Digital Object Identifier (DOI): doi:10.1016/j.virusres.2013.02.008

Publisher's Link: http://www.sciencedirect.com/science/article/pii/S0168170213000580

This item was retrieved from the K-State Research Exchange (K-REx), the institutional repository of Kansas State University. K-REx is available at http://krex.ksu.edu 
1 Genetic characterization and phylogenetic analysis of Skunk-Associated

2 Rabies Viruses in North America with special emphasis on the Central Plains

4 Rolan Davis ${ }^{1}$, Susan A. Nadin-Davis ${ }^{2}$, Michael Moore ${ }^{2}$ and Cathleen Hanlon ${ }^{1}$

6 Kansas State University Rabies Laboratory, 2005 Research Park Circle, Manhattan, KS 66502,

$7 \quad$ USA $^{1}$; Animal Health Microbiology Research, Ottawa Laboratory (Fallowfield), Canadian Food

8 Inspection Agency, Ottawa, Ontario, K2J 4S1, Canada ${ }^{2}$

9

10

11 * communicating author R. Davis, Email rdavis@vet.k-state.edu

12

13

14 Key words: rabies virus, phylogeography, phylogeny, nucleoprotein, glycoprotein 


\section{Abstract}

16 Across North America the skunk acts as a reservoir for several rabies virus variants. Some of

17 these variants are geographically restricted in range as is the case for the California skunk variant

18 and two distinct variants present in Mexico. In contrast the North Central and South Central

19 skunk rabies viruses are dispersed in overlapping ranges over large areas of the Midwestern

20 region of the United States with the former extending into southern parts of the Canadian

21 prairies. Despite this extensive range, there has been only very limited molecular characterization

22 of these two viral variants. This study has examined the genetic diversity of the rabies viruses

23 associated with North American skunks, with particular emphasis on the South Central skunk

24 variant which was found to comprise three distinct geographically restricted groups of viruses

25 that could in some cases be further sub-divided. The phylogenetic relationships of these groups

26 and sub-groups allowed us to infer the likely direction of spread of these variants in some

27 instances. Patterns of amino acid replacement of North American skunk-associated rabies viruses

28 for both the nucleoprotein and glycoprotein products are also examined. These patterns reflect

29 the virus phylogeny but no amino acid residues associated specifically with the skunk host were 30 identified. 


\section{Introduction}

Rabies virus is the prototype species of the Lyssavirus genus (ICTV, 2011). Despite its small $12 \mathrm{~Kb}$ single-stranded negative sense RNA genome and limited five gene coding capacity

34 (Wunner, 2007), infection with this virus results in rabies, a feared neurological disease that is 35 universally fatal except in extremely rare circumstances (Jackson, 2007). These viruses can

36

37 infect any mammalian species but they are maintained in association with particular reservoir hosts (Hanlon et al., 2007). Although both human and animal disease can be prevented through time-tested vaccination and post-exposure prophylactic regimens, these viruses continue to present a risk of disease around the world today, even in many developed countries.

Prior to the advent of animal control measures coupled with effective vaccination campaigns, the main public health threat in the United States was primarily from canine rabies virus variants transmitted by domestic dogs. As canine rabies virus variants were driven to extinction in the US, public awareness of the role of wildlife in rabies transmission increased (Price et al., 1961) and, by 1960, diagnosed cases in wildlife grew to outnumber cases in domestic animals(Eng et al., 1989). Skunks were the most commonly diagnosed species in the United States during the years 1961 to 1989, but then were superseded in the 1990s by the raccoon rabies epizootic in the mid-Atlantic and north-eastern states (Blanton et al., 2009).

In the United States, rabies cases among skunks were first reported in California as early as 1826(Hovey, 1874). More widespread epizootics began to emerge in the late 1950s with four distinct regions being affected by 1960 (Charlton et al., 1975). These regions included: 1 ) the north-central states and southern regions of the Canadian-prairie Provinces, 2) Texas in the south, 3) California in the west, and 4) north-eastern states neighboring the Canadian provinces 
53 of Ontario and Quebec. Following the advent of monoclonal antibody panel typing (Smith et al.,

54 1986), the distinctive nature of the viruses responsible for these outbreaks was established and

55 later confirmed by molecular epidemiological studies (Nadin-Davis et al., 2002). The viruses

56 responsible for these outbreaks are currently referred to as the North Central skunk (NCSK), the

57 South Central Skunk (SCSK) and the California skunk (CASK) variants respectively while

58 skunks in Ontario and New York State were shown to harbor viruses similar to the arctic fox

59 type spread by red foxes in the same region, although it has been proposed that the skunk acts as

60 a secondary host for this variant (Nadin-Davis et al., 2006). These variants represent no less than

61 three of the seven major rabies virus lineages identified world-wide (Bourhy et al., 2008). NCSK

62 and CASK are branches of the cosmopolitan lineage thought to have been spread from Europe to

63 many parts of the world during the colonial period, the SCSK variant is a member of the

64 American Indigenous lineage found only on that continent, and the arctic fox variant is a member

65 of the Arctic/Arctic-like lineage which circulates in northern climes and across large parts of

66 Asia (Nadin-Davis et al., 2007, 2012; Kuzmin et al., 2008). In the US and Canada, the striped

67 skunk (Mephitis mephitis), which is the principal skunk species diagnosed with rabies, is

68 believed to be the maintenance host for these three skunk-associated rabies epizootics although

69 the disease has been documented in other species such as hog-nosed (Conepatus leuconotus) and

70 hooded skunks (Mephitis macroura) (Hass and Dragoo, 2006). In Mexico, additional viral

71 strains associated primarily with the spotted skunk (Spilogale putorius) have been identified

72 Aranda and Lopez-de Buen, 1999; Velasco-Villa et al., 2002; Nadin-Davis and Loza-Rubio, 73 2006).

Although the last reported human death due to skunk transmitted rabies occurred in the

75 United States in 1981 (Krebs et al., 2000), cases of infected domestic animals help to underscore 
the public health importance of skunk transmitted rabies virus variants (Rupprecht et al., 1995).

77 Thus while skunks are the predominant reservoir species across the US Midwest, with 56\% of reported cases from these states occurring within skunks in 2008 (Blanton et al., 2009), cases within domestic species, presumably caused by skunk transmitted rabies, accounted for another $14 \%$ of reported cases. Rabies cycles through the skunk population of the Great Plains with regular peaks and troughs in the number of reported cases (Pool and Hacker, 1982; Oertli et al., 2009). The factors that define these peaks are not well understood but it is speculated that rabies cases may be directly tied to fluctuations in the skunk population density. Indeed cyclical patterns of rabies cases have been observed in wildlife species elsewhere. In Ontario, fox rabies incidence patterns of varying periodicity defined several discrete geographical units differing in host species distribution and persistence (Tinline and MacInnes, 2004); it was speculated that host meta-population structure plays a key role in disease persistence. In Europe, fox population density, turnover and social interactions were identified as the most important ecological factors influencing disease patterns (Steck and Wandeler, 1980). A detailed description of the molecular epidemiology of the rabies viruses circulating in skunks across the Great Plains was expected to reveal useful information about the spread of the disease in this population that is crucial to planning and implementation of effective rabies control and prevention strategies directly in this reservoir species. However, prior to the start of this study there was insufficient viral gene sequence data available to support such analysis, as relatively few examples of genomic sequences from skunk rabies viruses had appeared in the literature (Nadin-Davis et al., 1997; Velasco-Villa et al., 2008) or were available in the public databases. To address this deficiency and generate the first detailed analysis of the phylogeography of the SCSK rabies virus variant, a substantial database of nucleotide sequences of skunk-associated rabies viruses from six states 
99 (Arkansas, Kansas, Missouri, Nebraska, Oklahoma and South Dakota) in the US Midwest has 100 been compiled. Sequence information for the $\mathrm{N}$ (nucleoprotein) gene (all samples) and for the G 101 (glycoprotein) gene (most samples) was generated since the targeting of these two genes for 102 phylogenetic studies allows comparison with many other sequences in the public databases; 103 moreover variation at the $G$ protein could be functionally significant with respect to host cell 104 binding, cell entry and pathogenesis (Wunner, 2007). This sequence information, when 105 combined with that from other skunk-associated rabies viruses, extends our knowledge of (1) the 106 level of genetic diversity, (2) phylogeny and (3) evolutionary processes operating on the proteins 107 of viruses that are associated with such a permissive, ubiquitous, and vagile host. 


\section{Materials and Methods}

111

\subsection{Samples}

The rabies-positive samples examined in this study, which originated from several US states, the prairie provinces of Canada and distinct regions of Mexico, were processed at two different laboratories. The 78 samples examined by the Kansas State University (KSU) Rabies Laboratory included 32 isolates from Kansas, 14 from Nebraska and a single sample each originally from Colorado and Florida, all collected during early 2009. Additional US samples were solicited from primary diagnostic facilities in the states of Arkansas (12), Missouri (four), Oklahoma (six) and South Dakota (six), with an additional two samples originating from Minnesota but provided by the facility in South Dakota. All 78 samples were received for routine diagnostic testing rather than active surveillance investigations and their designations were generated thus: first a two letter code to indicate state of origin followed by a two letter host species code and a two digit number indicating year of submission and finally a four digit submission number. Full details of these samples are provided in supplementary material (Table S1). The ZIP code from where the submission originated was recorded and mapped.

For comparative analysis, an additional 22 rabies-positive samples were characterized at the Ottawa laboratory (OLF); this included 14 CASK variant specimens from California and a sample from a Mexican skunk (designated by $\mathrm{V}$ followed by a three digit number and the variant type), and an additional seven samples from Western Canada, representing the northernmost range of the NCSK variant, that are designated by L followed by a six digit number, that includes the year of isolation (two digits) and a submission number (four digits), and the suffix WSK. Details of these samples, together with all other isolates accessed through GenBank and used for phylogenetic analyses, are listed in supplementary material (Table S2). 
2.2. RNA extraction, Reverse Transcription and PCR

Rabies-positive samples were stored at $-70^{\circ} \mathrm{C}$ until processed for RNA extraction. Approximately 5-10 mg of brain material was added to $100 \mu \mathrm{L}$ of a lysis buffer $(10 \mathrm{mM}$ TRIS $\mathrm{HCl}, 150 \mathrm{mM} \mathrm{NaCl}, 1.5 \mathrm{mM} \mathrm{MgCl} 2$ and $0.65 \%$ NP40 substitute) to rupture the cells, $1 \mathrm{~mL}$ of TRIzol reagent (Invitrogen, Carlsbad, CA) was added and the sample refrigerated overnight ( 18 hours). Lysates were then processed according to the instructions provided by the manufacturer of the TRIzol reagent and each final dried RNA pellet was re-suspended in $100 \mu \mathrm{l}$ of RNase-free water and stored at $-70^{\circ} \mathrm{C}$.

For those samples processed at the KSU rabies facility, amplification of the viral genome at both the nucleoprotein $(\mathrm{N})$ and the glycoprotein $(\mathrm{G})$ genes was performed by generating two overlapping amplicons for each gene using the collection of primers described in supplementary material (Table S3). One of four oligonucleotide primers was annealed to the viral RNA target in a reaction that contained $5 \mu \mathrm{l}$ of the purified RNA and $1 \mu \mathrm{l}$ of reverse transcription (RT) primer (5 $\mu \mathrm{M} / 10 \mu \mathrm{M}$ for degenerate primers). Mixtures were heated to $94^{\circ} \mathrm{C}$ for 90 seconds and then cooled quickly on ice. Tubes received $14 \mu \mathrm{l}$ of a RT reaction buffer mix containing $100 \mathrm{mM}$ Tris (pH 8.3), $10 \mathrm{mM} \mathrm{MgCl,} 0.5 \mathrm{mM}$ each dNTP, 10 units of reverse transcriptase and 16 units RNase inhibitor (both produced by Roche Diagnostics, Indianapolis, IN). Reactions were incubated at $42^{\circ} \mathrm{C}$ for 90 minutes. Following RT, $80 \mu$ of PCR pre-mix was added to the $20 \mu \mathrm{l}$ RT product. The pre-mix contained $69 \mu \mathrm{l}$ of distilled water, $8 \mu \mathrm{l}$ of Tris ( $1 \mathrm{M}, \mathrm{pH} 8.3), 0.5 \mu \mathrm{l}$ AmpliTaq DNA polymerase (Applied Biosystems, Branchburg, NJ), $1 \mu$ l of forward primer (20 $\mu \mathrm{M} / 40 \mu \mathrm{M}$ for degenerate primers) and $1.5 \mu \mathrm{l}$ of reverse primer $(20 \mu \mathrm{M}) / 40 \mu \mathrm{M}$ for degenerate primers. Thermal cycling was performed in a Thermo PxE 0.2 with an initial denaturation at $94^{\circ} \mathrm{C}$ for 60 seconds, followed by 39 cycles of $94^{\circ} \mathrm{C}$ for 30 seconds, $42^{\circ} \mathrm{C}$ for 30 
156 seconds, $72^{\circ} \mathrm{C}$ for 90 seconds followed by a final cycle ending with a seven-minute elongation 157 period at $72^{\circ} \mathrm{C}$.

For those samples processed at the OLF rabies facility, RT-PCR of the rabies virus N

159 gene was performed essentially as described previously (Nadin-Davis, 1998) except that primer

160 RabN1 was replaced by primer RVfor2, (5'-gtACGCTTAACAACAARAYCARAGAA-3'

161 targeting bases 1-24 at the 3' end of the genomic RNA) for both the RT and PCR. RT-PCR of

162 the G gene was described previously (Nadin-Davis et al., 1997, 1999).

\section{2.3. PCR product purification and sequencing}

Each PCR was screened for successful amplification by analysis of an aliquot by standard agarose gel electrophoresis. Rabies virus-specific amplicons were recovered from the remaining

166 PCR product using a Wizard PCR Prep DNA Purification System as recommended by the 167 manufacturer Promega (Madison, WI) either directly (OLF) or after size fractionation by 168 electrophoresis through 2\% NuSieve low melting point agarose (KSU).

171 (AGTC), Lexington, KY. Products prepared at OLF were sequenced in-house using a NEN

172 4200L automated sequencing system (LiCor Biosciences, Lincoln, NE) with IR-dye labeled

173 primers and a SequiTherm EXCEL ${ }^{\mathrm{TM}}$ II DNA sequencing kit (Epicentre Biotechnologies)

174 obtained from Interscience, ON.

\section{2.4. Sequence Analyses}

Sequences received from the AGTC facility were aligned and edited using Bioedit (Hall,

177 2011). Sequences generated at OLF were compiled and edited using Eseq version 3.0 (LiCor

178 Biosciences). Final sequence databases of complete coding regions for the $\mathrm{N}$ gene (1350 bases), 
179 G gene (1572 bases) or the concatenated data from both genes (2922 bases) were aligned using 180 CLUSTALX version 1.8 (Thompson et al., 1997). MEGA version 4 (Tamura et al., 2007) was 181 used for generation of phylogenies using the neighbor joining (NJ) and maximum parsimony 182 (MP) methods, for computation of transition/transversion ratios and 183 synonymous/nonsynonymous nucleotide substitution rates (dN/dS) using the Kumar method and

184 for translation of nucleotide sequences to protein. Modeltest (Posada and Crandall, 1998) was 185 used to identify the General Time Reversible gamma model with invariant sites $(G T R+G+I)$ as 186 the generally applicable nucleotide substitution model that best fit both the $\mathrm{N}$ and $\mathrm{G}$ gene 187 sequence databases and this model was used for generation of maximum likelihood (ML) 188 phylogenies by PhyML, version 3.0 (Guindon et al., 2010).

189

190

191

192

193

194

195 196

197

\section{Results}

\subsection{Phylogeography of skunk rabies viruses in the Midwestern US}

Initial efforts focused on full length $\mathrm{N}$ gene analysis, but since these data yielded phylogenies that were only modestly supported, additional studies targeting the G gene were undertaken. Analysis was completed on 59 unique $\mathrm{N}$ sequences, on 64 unique $\mathrm{G}$ sequences and 68 unique sequences when N \& G were concatenated as previously described (Kuzmin et al., 2010); this database included a newly generated sequence from an isolate of raccoon variant (FLRC090148).

Results of a neighbor joining (NJ) analysis for these 68 concatenated $\mathrm{N}$ and $\mathrm{G}$ sequences and an EBLV2 isolate included as an out-group to the rabies virus clade are shown in Figure 1. Phylogenetic trees generated by analysis of each individual coding region all exhibited a similar 
201 topology; however, several lineages were more highly supported when both genes were analyzed 202 in tandem rather than individually (data not shown). Samples represented both skunk transmitted 203 viral variants circulating in the central plains. All samples from South Dakota and Minnesota 204 carried the NCSK variant together with a single outlying sample isolated from a dog from 205 Arkansas. The remaining 11 samples from Arkansas along with all samples from Kansas, 206 Missouri, Nebraska and Oklahoma were members of the SCSK variant. Neighbor-joining 207 analysis identified considerable diversity within this group and delineated three strongly 208 supported clades (Figure 1). The most outlying clade (SCSK I), is comprised entirely of samples 209 from Oklahoma. Clade SCSK II is entirely populated by isolates from Arkansas and Missouri 210 with well supported sub-division into IIA and IIB types. A third clade (SCSK III), made up of 211 samples primarily distributed across Kansas and Nebraska, is further subdivided into two types

212 with some samples from Kansas (IIIA) clearly differentiated with strong support from the 213 remaining members of the clade (IIIB). As illustrated in Figure 2, each of these clades occupies a 214 distinct geographical range with no observed spatial overlap of these viral types.

\subsection{Phylogenetics of North American skunk rabies viruses}

To place these results into a broader context additional phylogenetic analysis was

217 undertaken on a broader dataset that included additional newly sequenced skunk rabies virus

218 samples from Canada and the state of California. To allow inclusion of the greatest diversity of

219 viral samples, including those from bats and other terrestrial sources available in GenBank (see

220 list in Table S2), only N gene sequences were employed but this yielded robust phylogenies due

221 to the degree of genetic variation across the dataset. Figure 3 illustrates the results of a

222 maximum likelihood (ML) analysis completed on 122 rabies virus N genes (1350 bases) using a

223 European bat lyssavirus type $2(\mathrm{EBLV}-2)$ as an outgroup; similar phylogenies were generated 
224 using NJ and maximum parsimony (MP) methods (data not shown). As expected the ML tree

225 clearly divides all samples into two main lineages with strong support. The NCSK, CASK and

226 South Baja California (SBC) skunk variants all fall within a large group previously identified as

227 the cosmopolitan lineage (Nadin-Davis et al., 2002). The American indigenous lineage clearly

228 segregates into two groups of viruses associated with bats, including the Arizona skunk (AZ SK)

229 variant recently derived from a bat reservoir (Leslie et al., 2006; Kuzmin et al., 2012), and

230 terrestrial species respectively with sub-division of the latter into clades defining the raccoon,

231 central Mexican skunk and the US SCSK variants. Phylogenetic analyses using a smaller dataset

232 of $\mathrm{G}$ gene sequences of representative viruses generated similar trees (data not shown).

\section{3.3. Substitution patterns for $N$ and $G$ genes}

The nucleotide substitution patterns observed for both genes were examined using data

235 from representative skunk-associated viruses but excluding the Arizona skunk variant. Overall

236 transition/transversion ratios were high at 5.0403 ( $N$ gene) and 4.6102 (G gene). N gene dN/dS

237 ratios for each variant within the dataset ranged from 0.0357 (SCSK) to 0.0925 (SBC skunk)

238 with other variants yielding intermediate values (Table 1). It is unclear if the relatively high

239 value for the SBC skunk variant is significant or if it is a consequence of the limited numbers of

240 isolates of this type examined. Values for $\mathrm{dN} / \mathrm{dS}$ ratios for the G gene tended to be 2-3 times

241 greater due to a higher level of non-synonymous substitutions at this less conserved locus. These

242 values overwhelmingly supported the operation of purifying selection on these genes $(p=0.0000$

243 for all groups analysed using the Kumar method in MEGA) rather than neutral or positive

244 selective evolutionary forces.

245 3.4. Coding differences within the $\mathrm{N}$ gene 
247 this study to deduced $\mathrm{N}$ protein coding sequences enabled a detailed comparison of this viral 248 protein. For all skunk-associated viral variants, excluding the Arizona skunk variant, pairwise N

249 protein distance values ranged from 0 to 0.08 , corresponding to amino acid differences ranging

250 between 0 and 34. An alignment of these nucleoprotein sequences identified many highly

251 conserved residues as well as some variable positions that appear to reflect the phylogenetic

252 relationships between these variants. Figure S1 (Supplemental data) shows such an alignment

253 using representatives of all the skunk variants together with sequences from a few other viruses

254 that circulate in other reservoir hosts as illustrated in Figure 3. The most notable coding

255 differences found within the skunk-associated rabies variants are identified in Figure S1 and

256 discussed further below.

257 3.5. Coding differences within the G gene

258 Analysis of the predicted glycoprotein for all skunk-associated viral types, excluding the

259 Arizona skunk variant, indicated pairwise distance values ranging from 0 to 0.17 , corresponding

260 to amino acid differences of between 0 to 79. A glycoprotein sequence alignment of skunk-

261 associated rabies viruses together with representatives of other rabies virus variants that circulate

262 in the central US (Figure S2) identified the 19 amino acid N-terminal signal peptide and the

263 hydrophobic trans-membrane domain (amino acids 439-461) as areas of high variability as

264 previously documented (Badrane et al., 2001). Differences between members of the

265 cosmopolitan lineage and the SCSK variant viruses were especially pronounced across these

266 regions. However substitutions at particular positions were retained within some variants and

267 viral types; the more notable are illustrated in Figure S2 and discussed further below. 


\section{Discussion}

Detailed molecular epidemiological studies of rabies viruses are increasingly providing insights into the emergence, history and transmission dynamics of rabies enzootics and epizootics (Holmes et al., 2002; Bourhy et al., 2008; Talbi et al., 2010). Since rabies has become entrenched within multiple wildlife species within North America, an understanding of how the virus was introduced and then spread within each host population may hold the key to control and eventual elimination of this disease. Rabies viruses within skunks have been noted for many decades and although phylogenetic studies have helped to trace the historical origins of the variants associated with this host (Velaco-Villa et al., 2008), a detailed phylogeographic study of the genomic diversity of skunk rabies variants within the central United States has not been published previously. This study has explored the diversity of rabies viruses associated with skunks across this region in which both NCSK and SCSK variants were found.

\subsection{SCSK rabies viruses}

Rabies case records suggest that the current SCSK variant emerged from a focus of skunk rabies cases in Texas in the mid 1950s, followed by subsequent spread of this outbreak throughout the southern Great-Plains region of the United States. While isolates of the SCSK variant virus recovered from the states of Arkansas, Oklahoma, Missouri, Kansas and Nebraska are not differentiated by classical antigenic methods, they do exhibit marked genetic diversity that allows their sub-division into three major viral clades (Figure 1). Significant clustering of isolates according to the state of submission was observed (Figure 2).

All viruses from across much of the states of Kansas and Nebraska collectively form the largest monophyletic grouping designated as clade III. Moreover, there is a well defined division 
290 between eight isolates labeled as IIIA which come primarily from eastern Kansas and the rest of 291 the samples (IIIB) of this clade. The identification of two viruses recovered from Colorado 292 (COSK090005) and Missouri (MOSK090041) which also group within clade IIIB suggest that 293 this type is responsible for the recent expansion of SCSK into these states, in particular in 294 Colorado where the epizootic has reached as far as the foothills of the Rocky Mountains. Isolate 295 OKBV090073 within this group is reported as occurring approximately 150 miles away from the 296 nearest isolate of the same type. The bovine host of this case had no history of travel so this viral 297 isolate likely represents a southern extension of type IIIB.

Clade II, comprised of samples from Arkansas and all but one of the samples from 299 Missouri, is also divided into two types (IIA and IIB) with strong support. The Arkansas viruses 300 of type IIB originate from the southern half of the state while the type IIA specimens originate 301 from the northern half of Arkansas and Missouri; the limited genetic variation of all samples 302 from Missouri, illustrated by their tight clustering within a branch of a much larger clade 303 dispersed across Arkansas, is consistent with spread of the disease northwards from neighboring 304 Arkansas. The distinct ranges of these two types are separated by the Arkansas River which may 305 serve as a barrier for transmission of viruses of this clade.

A small group of viruses, all originating exclusively from Oklahoma, comprised the 307 outlying clade I of the SCSK variant. This was the only variant recovered from this state with the 308 exception of specimen OKBV090073 that appears to represent an incursion of clade IIIB. Some 309 historical samples from Texas and New Mexico included in a broader phylogenetic analysis also 310 clustered as outliers of the SCSK variant, while additional samples from Texas segregated on 311 distinct branches within other parts of the SCSK clade (Figure 3). The oldest characterized virus 
312 of the SCSK variant, from a skunk recovered in Texas in 1968, appears to have a common

313 ancestry with clade III.

While the geographically distinct ranges of SCSK types are a unique finding of the

315 present study this was unexpected since a recent study of striped skunks within the Midwestern

316 United States showed that gene flow is high between animals from the Dakotas through to

317 Oklahoma (Barton et al., 2010). Accordingly admixture of viral biotypes should be observed.

318 However, the molecular epidemiology described in the present study, albeit from a limited time

319 period, seems to show that such mixing of viral types is not the case. These findings may

320 indicate that the viral variants move across the landscape in wave-fronts with localized genetic

321 drift leading to emergence of sub-types in particular areas. Alternatively it might indicate

322 adaptation of various biotypes to distinct habitats across the region although to date no evidence

323 for such evolutionary factors exists. As this sample set has demonstrated only a snapshot of sub-

324 type distribution across the landscape further retrospective and prospective studies will explore

325 temporal changes to this pattern and help to better understand the contributing factors.

\section{4.2. NCSK rabies viruses}

327 The epizootic due to the NCSK variant was first recognized in the late 1940s; Missouri reported 32828 cases of rabies within skunks in 1959, apparently due to spread of an epizootic front moving 329 south from the Dakotas (Parker, 1975). All South Dakota and Minnesota terrestrial isolates 330 examined in this study were exclusively of the NCSK variant. They clustered closely with the 331 Canadian samples recovered from the provinces of Saskatchewan and Manitoba that are located 332 directly north of the states of Montana, North Dakota and Minnesota. Indeed this sample set was 333 relatively homogeneous with no strongly supported phylogenetic structure, indicating that the 
334 virus in this border region has probably experienced little impediment to its spread across the

335 landscape. Especially notable is the limited variation observed within the Canadian specimens

336 despite inclusion of samples recovered over a 13 year period. However, viruses from further

337 afield exhibited greater diversity. For example, sample ARDG090042, recovered from a dog in

338 north-eastern Arkansas in 2009, was an outlier to this group based on analysis of both N and G

339 gene sequences, as were samples from Kentucky and Wisconsin (Figure 3). Indeed previous

340 samples from this region of north-eastern Arkansas and south-eastern Missouri show a pocket of

341 NCSK circulating among skunks in the region (unpublished data). Current national data (Blanton

342 et al., 2010) show a southern extension of this variant within central Kentucky and Tennessee but

343 not as far west as Arkansas and Missouri. Regardless, the single Arkansas isolate evaluated here

344 is not from an area contiguous with the more northern regions affected by this viral variant and

345 its divergence is thus not unexpected. Analysis of more samples from states where this rabies

346 virus variant occurs is needed to complete our understanding of its range and genetic diversity.

\section{4.3. CASK rabies viruses}

Prior studies on rabies in California have used antigenic typing tools and genetic methods based on PCR and restriction endonuclease analysis to explore the diversity of the virus

350 circulating in terrestrial species in the state (Crawford-Miksza et al., 1999). Subsequently

351 nucleotide sequence analysis has been undertaken on a limited number of isolates (Velasco-Villa

352 et al., 2008) but without the benefit of detailed spatial information on the source of those isolates

353 so as to allow correlation with the earlier studies. This study genetically characterized a small

354 set of viruses from terrestrial species from different regions of California to allow comparison

355 with other skunk-associated viruses and to explore their regional variation. Only the northern

356 half of the state was represented in this sample set (see Figure 4) since skunk rabies is rarely if 
357 ever reported in the southern counties (Crawford-Miksza et al., 1999). All California viruses

358 formed a monophyletic clade (CASK) which can readily be sub-divided into three types that

359 exhibit geographical localization. Furthermore the identification of several amino acid coding

360 differences between these viruses support the conclusion that these three types represent some of

361 the discrete antigenic types proposed previously (Crawford-Miksza et al., 1999). CASK type a

362 comprises isolates from Mariposa county (San Joachim valley variant); type b consists of

363 specimens from the north-eastern region of the state including the Sonoma/North coast regions

364 and some inland areas (Trinity and Yolo counties); type c from Glenn, Sutter, Colusa and

365 Amador counties corresponds to the Sacramento Valley variant which was previously described

366 as being particularly distinctive with respect to its monoclonal antibody binding pattern

367 (Crawford-Miksza et al., 1999).

4.4. Mexican skunk rabies viruses

Included in our analysis are seven rabies isolates from Mexico that segregate into two discrete clades representing variants localized to South Baja California (SBC skunk) and central

371 Mexico, also referred to elsewhere as the MEXSK-2 and MEXSK-1 variants respectively

372 (Velasco-Villa et al., 2008). These variants circulate predominantly in spotted skunks and

373 possibly also hog-nosed skunks. The SBC skunk variant is closely related to the CASK variant,

374 perhaps not surprising given the geographical proximity of the areas where they circulate, while

375 the central Mexican variant clusters as an outlying group to both the SCSK and raccoon strains.

376 The predominant role of spotted skunks in this enzootic may be significant. During the mid $19^{\text {th }}$

377 century when rabies transmitted by skunks, or Rabies Mephitica as it was designated (Hovey,

378 1874), was common in Kansas and Colorado, both the spotted skunk and the striped skunk were

379 responsible. While historically the spotted skunk apparently played a significant role in disease 
transmission, today this species is nearly extirpated from most of its historical range in the central United States and all of the recent US isolates detailed here are from striped skunks. We speculate that the viruses circulating currently in central Mexican spotted skunks are remnants of the virus that predominated in the Great Plains over 150 years prior and gave rise to the SCSK variant. Moreover, the position of the raccoon variant within this cluster of skunk-associated viruses is consistent with the hypothesis that the raccoon strain emerged after a host shift from a skunk-associated virus rather than directly from a bat reservoir. Indeed all bat-associated viral variants appear to group well outside of the cluster of viruses associated with terrestrial hosts. In contrast, the recently emerged Arizona skunk variant, known to have arisen by host shift events from a bat reservoir, clusters closely with the responsible big brown bat variant (Leslie et al., 2006; Kuzmin et al., 2012).

\subsection{Viral evolutionary processes}

The database of $\mathrm{N}$ and $\mathrm{G}$ gene sequences generated in this study showed that the patterns of nucleotide substitution exhibited by skunk-associated rabies viruses are similar to those observed in prior studies on lyssavirus diversity in general (Bourhy et al., 2008; Delmas et al., 2008). Changes are predominantly synonymous in nature and most nonsynonymous mutations result in very conservative amino acid substitutions. However, this study did identify some amino acid substitutions that are associated with particular viral variants or clades. Within the $\mathrm{N}$ gene amino acid replacements of particular note are at the following positions: residues specific to all members of the SCSK variant occur at positions 3 (Thr in place of Ala), 93 (Asp in place of Gly), and 448 (Asn in place of Ser); replacement of Met by Leu at position 126 in SCSK and the Central Mexican variants; differences specific to the SCSK III variant at residue 209 (Ala in place of Thr), to the SCSK IIIB variant at amino acid 135 (Gln in place of Pro), and at position 
182 (Ile in place of Val) for both SCSK variants II and III. Several amino acids were represented

404 422 protein sequence.

at residue 40 with either Cys or Ser predominating in most variants while Gly was restricted to SCSK IIa and III; distinct substitutions at residues 254 (Lys) and 428 (Gly) observed in the SCSK IIb variant further reinforced the distinctive nature of this viral group. The SBC skunk viruses exhibited distinctive amino acid residues at positions 11 (Tyr), 36 (Ser), 84 (Ile) and 407(Ala) while the Central Mexican viruses were distinctive at residues 99 (Gln), 181 (Val) and 388 (Asp). Interestingly, with just a few exceptions, several coding differences between the CASK variants were observed. Specific residues were associated with variants "a” at positions 40 (Phe), 128 (Met) and 410 (Ile), with variants “b” at residues 9 (Arg except for V640) and 84 (Pro) and with variants “c” at positions 13 (His), 202 (Ser) and 255 (Asp) while a His residue was located at position 369 for variants "b" and “c", a substitution also shared with samples V212TXSK and 2311Mxzacsk01 (central Mexican skunk). As a group, the NCSK samples exhibited much less variability within the nucleoprotein with the exception of the more outlying samples such as ARDG090042, 421Kydg07 and 3789SK. These three samples individually and collectively exhibited a number of amino acid replacements (e.g. at residues 126 and 433) compared to other members of the NCSK clade. However many of these substitutions were also observed in viruses of the other clades examined suggesting that these positions had sufficient flexibility to allow genetic drift. The Arizona skunk variant retained the coding capability of the original bat variant and there was no evidence of adaptation to a skunk variant within the $\mathrm{N}$

Within the G gene the following substitutions are of particular note: at amino acids 3739, a glycosylation site within antigenic site II was conserved in all members of the cosmopolitan lineage but not in many SCSK isolates nor in any bat-associated variants; moreover a Ser-Thr 
426 substitution at position 201 also within antigenic site II was found in all members of the SCSK

427 III clade; the core sequence of the conserved linear G5 epitope, “HDFR” at residues 261-264

428 (Cai et al., 2010), was substituted to HDLH in all SCSK variants and indeed in this sample set

429 only the first two amino acids of this epitope were conserved. In contrast the linear epitope

430 “WXXXDI” at residues 14-19 (Mansfield et al., 2004), represented here as WSPIDI, was highly

431 conserved together with its flanking sequences. While most skunk-associated viruses were

432 conserved across antigenic site III (residues 330-338), some variation occurred around this site,

433 most notably in bat-associated variants where residue 333 was either the more common Arg or in

434 some cases Lys; however, either residue at this site maintains viral virulence (Tuffereau et al.,

435 1989). The distinctive Arkansas sample (ARDG090042) had 10 unique substitutions in the

436 glycoprotein compared to other US members of the NCSK variant. Several other substitutions

437 observed only in particular viral variants are identified in Figure S2. As noted for the

438 nucleoprotein analysis, the viruses of the Arizona skunk variant exhibit glycoprotein sequences

439 highly characteristic of the bat viral variant from which they are derived and are quite distinctive

440 from the SCSK viruses described in this report.

These changes appear most likely to have arisen through chance mutation followed by

442 fixation in the absence of selective pressure. No amino acids specific to the skunk-associated

443 viruses and distinctive from the viruses of other hosts were identified in this study in either the $\mathrm{N}$

444 or G proteins and it would thus appear that these products are unlikely to harbor residues that

445 confer host specificity, a finding consistent with that reported by others (Velasco-Villa et al.,

446 2008). Indeed, it has been proposed that high levels of synonymous substitutions may allow

447 rabies virus to become "pre-adapted to replicate in a wide range of species” (Holmes et al., 2002;

448 Gordon et al., 2004; Velasco-Villa et al., 2008). However, the variation in these gene and protein 
449 sequences documented here may have practical utility for the development of virus sub-typing

450 methods employing either monoclonal antibodies or molecular-based methods directed to these

451 variable sites. Such tools would allow variant and sub-type tracking to facilitate further

452 epidemiologic analysis of the spread of these viruses across the landscape. Moreover, given that

453 the striped skunk appears to be relatively susceptible to being infected by rabies, as well as

454 permissive to maintaining the virus as a reservoir host, continued epidemiologic vigilance is

455 warranted to permit early detection of future host jumps of other rabies virus variants into this

456 species.

\section{4.6. Conclusions}

458 In summary, this study has shown that rabies virus variants associated with North American

459 skunk populations have emerged from two distinct ancestral sources and are subject to purifying

460 selection which significantly restricts genetic drift. There is no evidence that viruses associated

461 with this host bear specific and unique amino acid residues in either the $\mathrm{N}$ or $\mathrm{G}$ proteins but

462 genetic signatures of both the CASK and SCSK variants identify sub-types with discrete

463 geographical ranges. Such information provides a baseline for subsequent molecular

464 epidemiological studies exploring the direction and speed of spread of the virus across the

465 landscape, information that may facilitate any future control efforts against this disease. 


\section{References}

Aranda, M. and Lopez-de Buen, L., 1999. Rabies in skunks from Mexico. J. Wildl. Dis. 35, 574577.

Badrane, H., Bahloul, C., Perrin, P., Tordo, N., 2001. Evidence of two lyssavirus phylogroups with distinct pathogenicity and immunogenicity. J. Virol. 75, 3268-3276.

Barton, H.D., Gregory, A.J., Davis, R., Hanlon, C.A., Wisely, S.M., 2010. Contrasting landscape epidemiology of two sympatric rabies virus strains. Mol. Ecol. 19, 2725-2738.

Blanton, J.D., Palmer, D., Rupprecht, C.E., 2010. Rabies surveillance in the United States during 2009. J. Am. Vet. Med. Assoc. 237, 646-657.

Blanton, J.D., Robertson, K., Palmer, D., Rupprecht, C.E., 2009. Rabies surveillance in the United States during 2008. J.Am.Vet.Med.Assoc. 235, 676-689.

Bourhy, H., Reynes, J.-M., Dunham, E.J., Dacheux, L., Larrous, F., Huong, V.T.Q., Xu, G., Yan, J., Miranda, M.E.G., Holmes, E.C., 2008. The origin and phylogeography of dog rabies virus. J. Gen. Virol. 8, 2673-2681.

Cai, K., Feng, J.-N., Wang, Q., Li, T., Shi, J., Hou, X.-J., Gao, X., Liu, H., Tu, W., Xiao, L., Wang, H., 2010. Fine mapping and interaction analysis of a linear rabies virus neutralizing epitope. Microbes Infect. 12, 948-955.

Charlton K.M., Webster, W.A., Casey, G.A., 1975. Skunk rabies. In: Baer, G.M. (Ed.), The Natural History of Rabies, Vol. 2. Academic Press, New York, pp. 307-324. 
Crawford-Miksza, L.K., Wadford, D.A., Schnurr, D.P., 1999. Molecular epidemiology of enzootic rabies in California. J. Clin. Virol. 14, 207-219.

Delmas, O., Holmes, E.C., Talbi, C., Larrous, F., Dachent, L., Bouchier, C., Bourhy, H., 2008. Genomic diversity and evolution of the lyssaviruses. PLoS One 3(4), e2057.

Eng, T.R., Hamaker, T.A., Dobbins, J.G., Tong, T.C., Bryson, J.H., Pinsky, P.F., 1989. Rabies surveillance, United States, 1988. MMWR CDC Surveill Summ. 38, 1-21.

Gordon, E.R., Curns, A.T., Krebs, J.W., Rupprecht, C.E., Real, L.A., Childs, J.E., 2004. Temporal dynamics of rabies in a wildlife host and the risk of cross-species transmission. Epidemiol. Infect. 132, 515-524.

Guindon S., Dufayard J.F., Lefort V., Anisimova M., Hordijk W., Gascuel O. 2010. New Algorithms and Methods to Estimate Maximum-Likelihood Phylogenies: Assessing the Performance of PhyML 3.0. Systematic Biol. 59, 307-21.

Hall, T.A., 2011. BioEdit: a user-friendly biological sequence alignment editor and analysis program for Windows 95/98/NT. Available at http://www.mbio.ncsu.edu/BioEdit/page2.html. Accessed August 14, 2012.

Hanlon, C., Niezgoda, M., Rupprecht, C. 2007. Rabies in Terrestrial Animals, In: Jackson, A.C., Wunner, W. (Eds.), Rabies. 2nd ed. Academic Press, London, UK, pp. 201-258.

Hass, C.C., Dragoo, J.W., 2006. Rabies in hooded and striped skunks in Arizona. J. Wildl. Dis. 42, 825-829. 
Holmes, E.C., Woelk, C.H., Kassis, R., Bourhy, H., 2002. Genetic constraints and the adaptive evolution of rabies virus in nature. Virology 292, 247-257.

Hovey, H.C., 1874. Rabies Mephitica. Am. J. Sci. Arts 7, 478-483.

International Committee on Taxonomy of Viruses. 2011. ICTV Files and Discussions. ICTV 2011 Master Species List - Version 1, February 21, 2012. Available at: http://talk.ictvonline.org/files/ictv_documents/m/msl/4090.aspx. Accessed March 7, 2012.

Jackson, A.C., 2007. Pathogenesis. In: Jackson, A.C., Wunner, W. (Eds.), Rabies. Academic Press, London, UK, p. 341-381.

Krebs, J.W., Smith, J.S., Rupprecht, C.E., Childs, J.E., 2000. Mammalian reservoirs and epidemiology of rabies diagnosed in human beings in the United States, 1981-1998. Ann. N. Y. Acad. Sci. 916, 345-353.

Kuzmin, I.V., Hughes, G.J., Botvinkin, A.D., Gribencha, S.G., Rupprecht, C.E., 2008. Arctic and Arctic-like rabies viruses: distribution, phylogeny and evolutionary history. Epidemiol. Infect. 136, 509-519.

Kuzmin, I.V., Mayer, A.E., Niezgoda, M., Markotter, W., Agwanda, B., Breiman, R.F., Rupprecht, C.E., 2010. Shimoni bat virus, a new representative of the Lyssavirus genus. Virus Res. 149, 197-210.

Kuzmin, I.V., Shi, M., Orciari, L.A., Yager, P.A., Velasco-Villa, A., Kuzmina, N.A., Streicker, D.G., Bergman, D.L., Rupprecht, C.E., 2012. Molecular inferences suggest multiple hosts shifts of rabies viruses from bats to mesocarnivores in Arizona during 2001-2009. PLoS Pathogens 8(6), e1002786. 
525 Leslie, M.J., Messenger, S., Rohde, R.E., Smith, J., Cheshier, R., Hanlon, C., Rupprecht, C.E., 2006. Bat-associated rabies virus in Skunks. Emerg. Infect. Dis. 12, 1274-1277.

527 Mansfield, K.L., Johnson, N., Fooks, A.R., 2004. Identification of a conserved linear epitope at 528 the N terminus of the rabies virus glycoprotein. J. Gen. Virol. 85, 3279-3283.

529 530 531 532 533 534 535 536 537 538 539 540 541 542

Nadin-Davis, S.A., 1998. Polymerase chain reaction protocols for rabies virus discrimination. J. Virol. Methods 75, 1-8.

Nadin-Davis, S.A., Loza-Rubio, E., 2006. The molecular epidemiology of rabies associated with chiropteran hosts in Mexico. Virus Res. 117, 215-226.

Nadin-Davis, S.A., Abdel-Malik, M., Armstrong, J., Wandeler, A.I., 2002. Lyssavirus P gene characterisation provides insights into the phylogeny of the genus and identifies structural similarities and diversity within the encoded phosphoprotein. Virology 298, 286-305.

Nadin-Davis, S.A., Huang, W., Wandeler, A.I., 1997. Polymorphism of rabies viruses within the phosphoprotein and matrix protein genes. Arch.Virol. 142, 979-992.

Nadin-Davis, S.A., Sampath, M.I., Casey, G.A., Tinline, R.R., Wandeler, A.I., 1999. Phylogeographic patterns exhibited by Ontario rabies virus variants. Epidemiol. Infect. 123, 325-336.

Nadin-Davis, S.A., Muldoon, F., Wandeler, A.I., 2006. Persistence of genetic variants of the arctic fox strain of Rabies virus in southern Ontario. Can. J. Vet. Res. 70, 11-19.

Nadin-Davis, S.A., Turner, G., Paul, J.P.V., Madhusudana, S.N., Wandeler, A. I., 2007. Emergence of Arctic-like rabies lineage in India. Emerg. Infect. Dis. 13, 111-116. 
545 Nadin-Davis, S.A., Sheen, M., Wandeler, A.I., 2012. Recent emergence of the Arctic rabies virus $546 \quad$ lineage. Virus Res. 163, 352-362.

547 Oertli, E.H., Wilson, P.J., Hunt, P.R., Sidwa, T.J., Rohde, R.E., 2009. Epidemiology of rabies in $548 \quad$ skunks in Texas. J. Am. Vet. Med. Assoc. 234, 616-620.

549 Parker, R.L. 1975. Rabies in Skunks, In: Baer, G.M. (Ed.), The Natural History of Rabies, Vol. 550 2. Academic Press, New York, pp. 41-51.

551 Pool, G.E., Hacker, C.S., 1982. Geographic and seasonal distribution of rabies in skunks, foxes $552 \quad$ and bats in Texas. J. Wildl. Dis. 18, 405-418.

553 Posada, D., Crandall, K.A., 1998. Modeltest: testing the model of DNA substitution. 554 Bioinformatics 14, 817-818.

Price, E.R., Blenden, D.C., Logue, J.T., 1961. Rabies in Missouri 1950-1960. Mol. Med. 58, 556 460-466.

557 Rupprecht, C.E., Smith, J.S., Fekadu, M.,, Childs, J.E., 1995. The ascension of wildlife rabies: a cause for public health concern or intervention? Emerg. Infect. Dis. 1, 107-114.

559 Smith, J.S., Reid-Sanden, F.L., Roumillat, L.F., Trimarchi, C., Clark, K., Baer, G.M., Winkler, 560 W.G., 1986. Demonstration of antigenic variation among rabies virus isolates by using 561 monoclonal antibodies to nucleocapsid proteins. J. Clin. Microbiol. 24, 573-580.

562 Steck, F., Wandeler, A., 1980. The epidemiology of fox rabies in Europe. Epidemiol. Rev. 2, 71563 96. 
Talbi, C., Lemey, P., Suchard, M.A., Abdelatif, E., Elharrak, M., Jalal, N., Faouzi, A., Echevarría, J.E., Morón, S.V., Rambaut, A., Campiz, N., Tatem, A.J., Holmes, E.C., Bourhy, H., 2010. Phylodynamics and human-mediated dispersal of a zoonotic virus. PLoS Path. 610: e1001166.

Tamura, K., Dudley, J., Nei, M., Kumar, S., 2007. MEGA4: Molecular Evolutionary Genetics Analysis (MEGA) software version 4.0. Mol. Biol. Evol. 24, 1596-1599.

Thompson, J.D., Gibson, T.J., Plewniak, F., Jeanmougin, F., Higgins, D.G., 1997. The CLUSTAL_X windows interface: flexible strategies for multiple sequence alignment aided by quality analysis tools. Nucleic Acids Res. 25, 4876-4882.

Tinline, R.R., MacInnes, C.D., 2004. Ecogeographic patterns of rabies in southern Ontario based on time series analysis. J. Wildl. Dis. 40, 212-221.

Tuffereau, C., Leblois, H., Bénéjean, J., Coulon, P., Lafay, F., Flamand, A., 1989. Arginine or lysine in position 333 of ERA and CVS glycoprotein is necessary for rabies virulence in adult mice. Virology 172, 206-212.

Velasco-Villa, A., Gomez-Sierra, M., Hernandez-Rodriguez, G., Juarez-Islas, V., MelendezFelix, A., Vargas-Pino, F., Velazquez-Monroy, O., Flisser, A., 2002. Antigenic diversity and distribution of rabies virus in Mexico. J. Clin. Microbiol. 40, 951-958.

Velasco-Villa, A., Reeder, S.A., Orciari, L.A., Yager, P.A., Franka, R., Blanton, J. D., Zuckero, L., Hunt, P., Oertli, E.H., Robinson, L.E., Rupprecht, C.E., 2008. Enzootic rabies elimination from dogs and reemergence in wild terrestrial carnivores, United States. Emerg. Infect. Dis. 14, 1849-1854. 
585 Wunner, W., 2007. Rabies Virus. In: Jackson, A.C., Wunner, W. (Eds.), Rabies. Academic Press, 586 London, UK, pp. 23-68.

587

588

589 
Figure legends

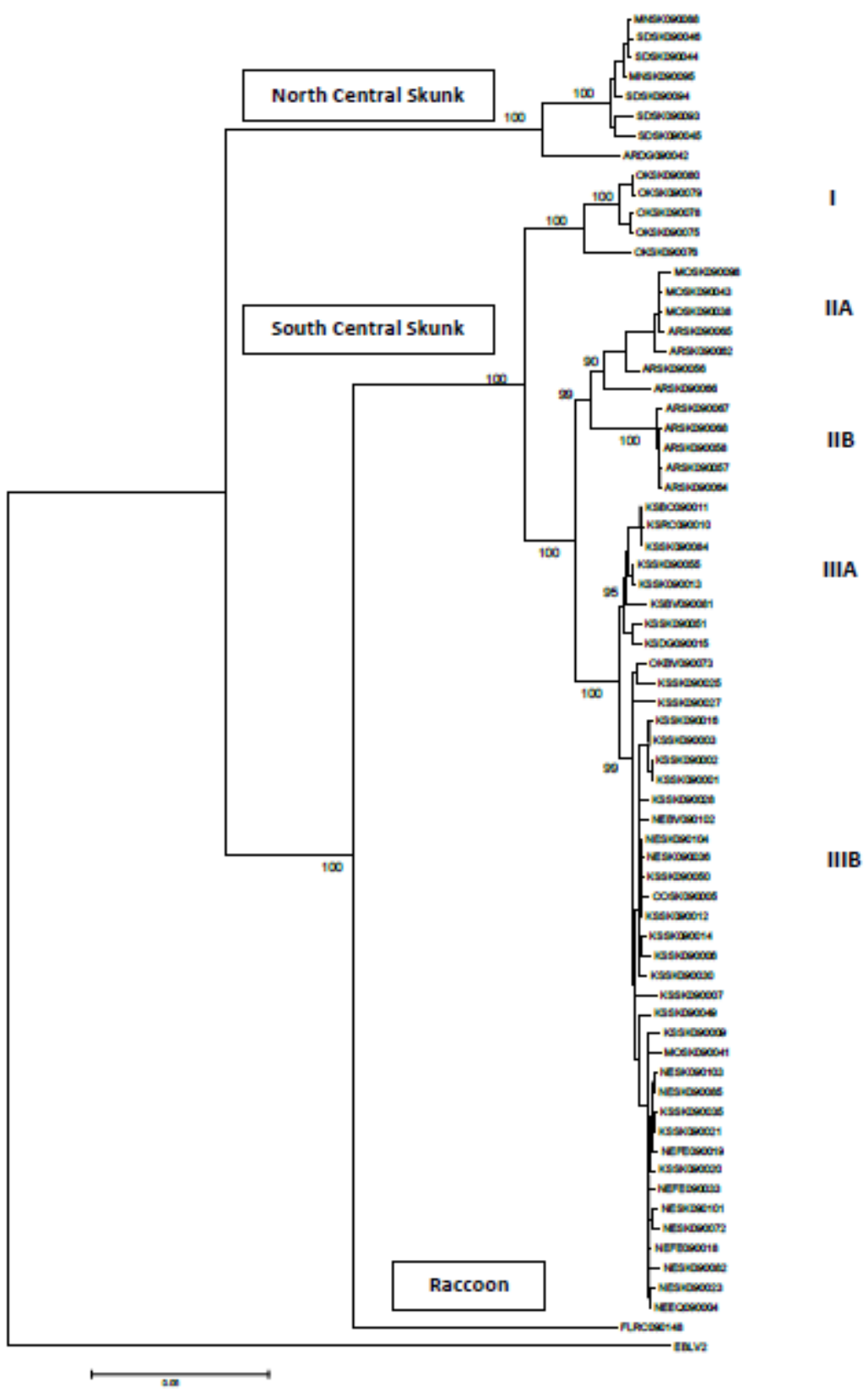

Figure 1. A phylogenetic tree of rabies virus sequences generated from 67 specimens infected with skunk variants. A NJ analysis was performed on concatenated N and $\mathrm{G}$ gene sequences; corresponding sequences for one raccoon virus variant and an EBLV2 isolate were included as outgroups. Bootstrap values for major branch points are shown within the tree. The names of the main variants are shown in boxes while the designations of the different clades and types of the SCSK variant as described in the text are provided to the right of the tree. A distance scale is shown at bottom left. 


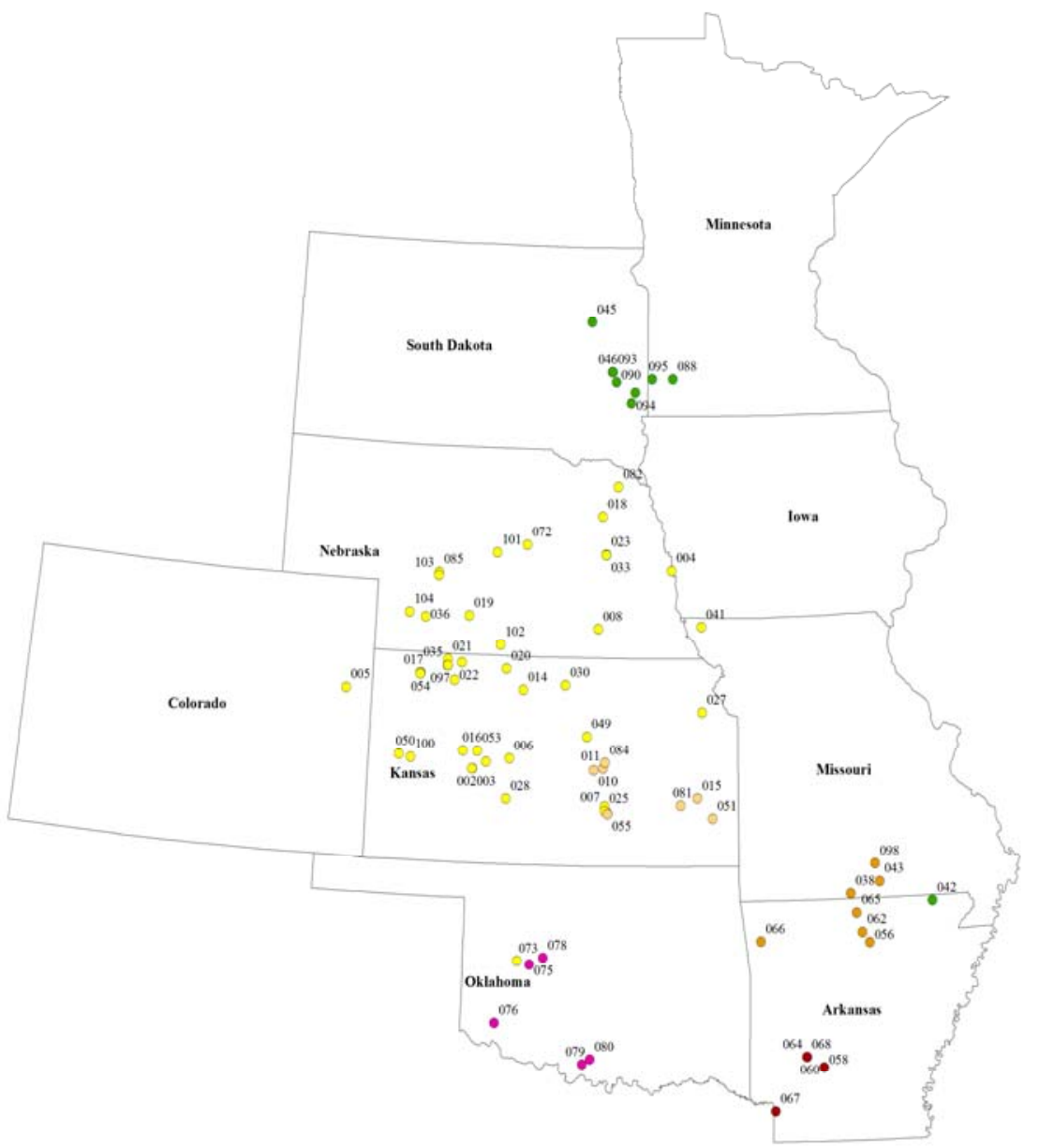

602

Figure2. Spatial distribution of all skunk variant isolates from the US Midwest examined in this study. Using ZIP code information each sample was mapped with a pin color coded based on the clade or type in which it clustered in the phylogenetic tree (Figure 1). Green pins represent the NCSK variant, while the SCSK variant clades are represented by pink (SCSK I), orange (SCSK IIA), red (SCSK IIB), peach (SCSK

607 IIIA) and yellow (SCSK IIIB). Samples are designated with the final 3 digits as detailed in Table S1.

608 
$\underline{\text { Figure } 3}$

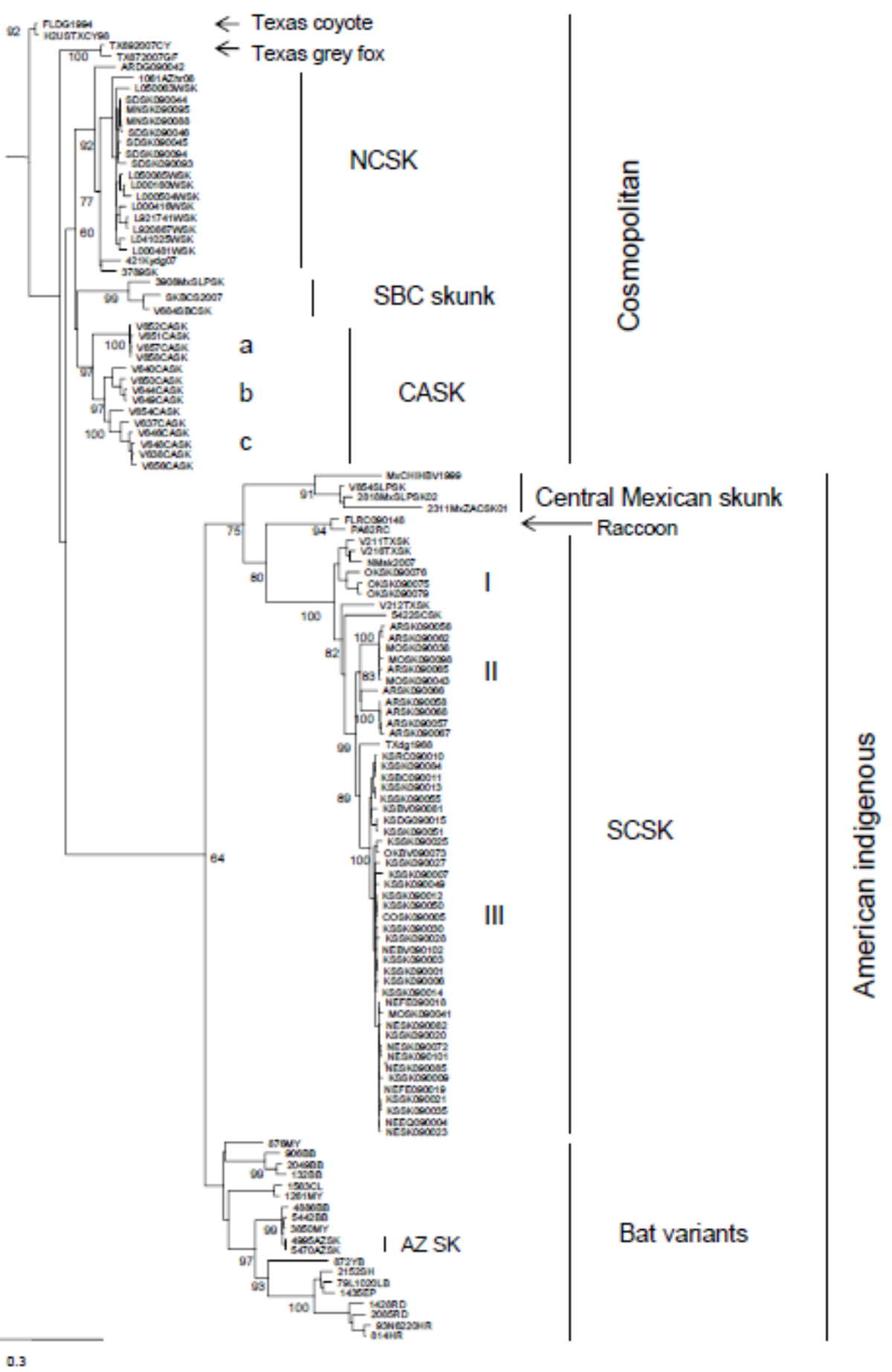

611 Figure 3. A maximum likelihood analysis of $\mathrm{N}$ gene sequences of North America skunk-associated rabies 612 viruses and representative isolates from other sympatric viral variants. The phylogenetic tree is rooted 613 to an EBLV2 outgroup (not shown). Bootstrap values for major branch points are shown in the tree. The 614 lineage variant type and type designations are shown to the right of the tree. A distance scale is shown 615 at bottom left. 
Figure 4

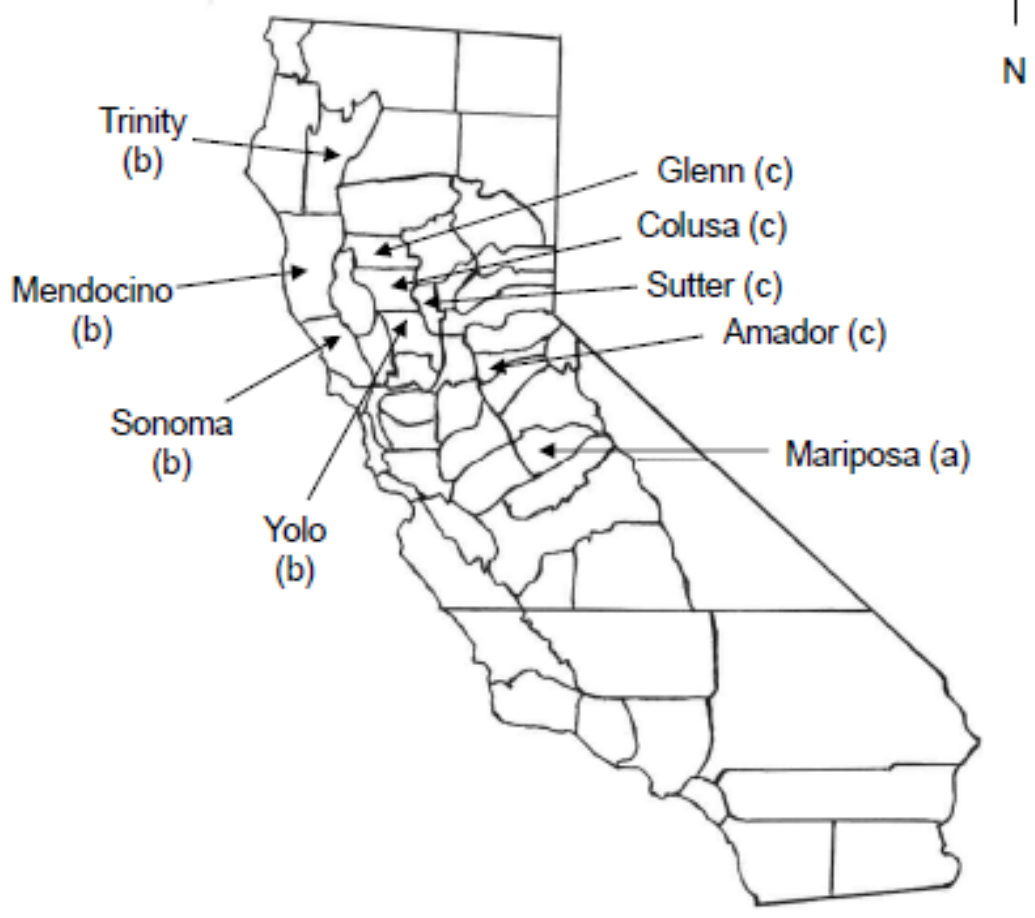

618 Figure 4. Map of the state of California showing the counties from which CASK rabies virus

619 variant isolates were characterized in this study. The viral types (a, b and c) identified by 620 phylogenetic analysis are indicated after the county name. 
622 Table 1

Table 1. Patterns of synonymous and nonsynonymous nucleotide differences between skunk-associated rabies viruses

\begin{tabular}{|c|c|c|c|c|c|c|c|c|}
\hline & \multicolumn{4}{|l|}{$\mathrm{N}$ gene } & \multicolumn{4}{|l|}{ G gene } \\
\hline & $\begin{array}{l}\text { No. of } \\
\text { samples in } \\
\text { group }\end{array}$ & dS & $\mathrm{dN}$ & $\mathrm{dN} / \mathrm{dS}$ & $\begin{array}{l}\text { No. of } \\
\text { samples in } \\
\text { group }\end{array}$ & dS & $\mathrm{dN}$ & $\mathrm{dN} / \mathrm{dS}$ \\
\hline NCSK & 20 & 0.0649 & 0.0041 & 0.0632 & 14 & 0.0645 & 0.0085 & 0.1318 \\
\hline CASK & 14 & 0.1399 & 0.0064 & 0.0457 & 2 & 0.2740 & 0.0352 & 0.1285 \\
\hline SCSK & 56 & 0.1037 & 0.0037 & 0.0357 & 58 & 0.0922 & 0.0108 & 0.1171 \\
\hline $\begin{array}{l}\text { Central } \\
\text { Mexico SK }\end{array}$ & 4 & 0.2295 & 0.0142 & 0.0619 & 1 & N/A & N/A & N/A \\
\hline SBC SK & 3 & 0.0951 & 0.0088 & 0.0925 & 1 & N/A & $\mathrm{N} / \mathrm{A}$ & N/A \\
\hline All variants & 97 & 0.5392 & 0.0144 & 0.0267 & 76 & 0.4529 & 0.0341 & 0.0753 \\
\hline
\end{tabular}

